Supplementary Information to:

\title{
Metal Seed Loss Throughout the Nanowire Growth: Bulk Trapping and Surface Mass Transport
}

\author{
Dany Chagnon ${ }^{\dagger}$ Eckhard Pippel, ${ }^{\star}$ Stephan Senz,${ }^{\dagger}$ and Oussama Moutanabbir ${ }^{\dagger}$ \\ †Département de Génie Physique, École Polytechnique de Montréal, Montréal, C.P. \\ 6079, Succ. Centre-Ville, Montréal, Québec, H3C 3A7 Canada \\ ${ }^{\ddagger}$ Max Planck Institute of Microstructure Physics, Weinberg 2, Halle, 06120 Germany
}



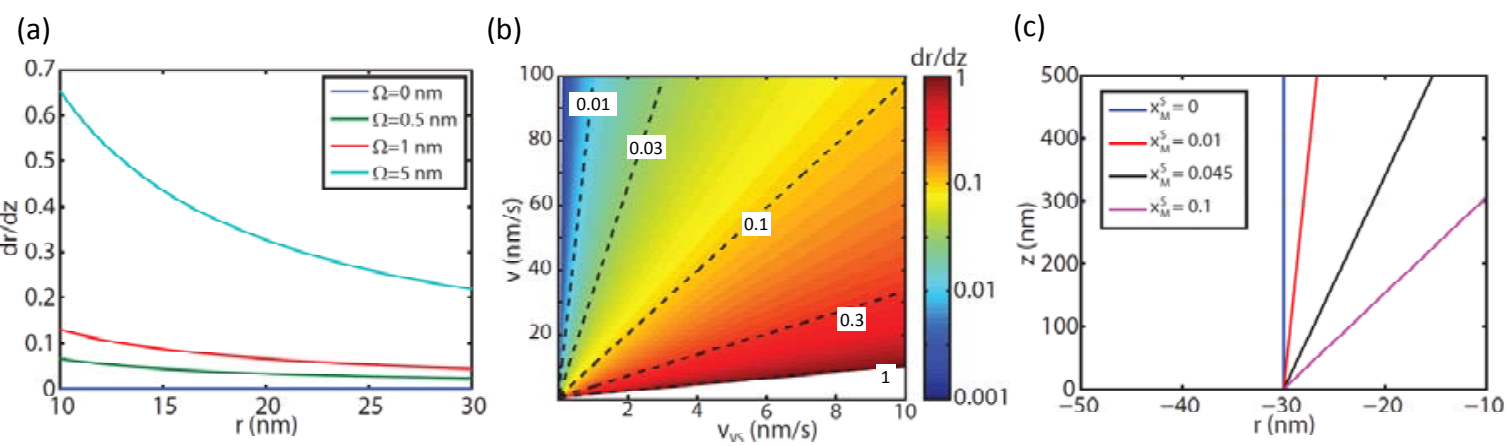

Figure S1: The influence of different phenomena on the nanowire morphology. The evolution of the nanowire tapering coefficient, $d r / d z$, as a function of the importance of sidewall diffusion (a) and vapor-solid growth rate (b). Note that the change in the nanowire radius induced by deposition on the nanowire sidewalls (homoepitaxy) is given by $d r / d z=v_{V S} / v$, thus increasing the growth velocity or decreasing the VS growth rate should obviously lead to straight nanowires. However, as indicated in the manuscript, under the nanowire growth conditions considered in this work, the VS growth rates are typically too low for the sidewall deposition to have any effect on the nanowire radius. (c) The evolution of the nanowire radius for different level of catalyst trapping in the nanowire. 


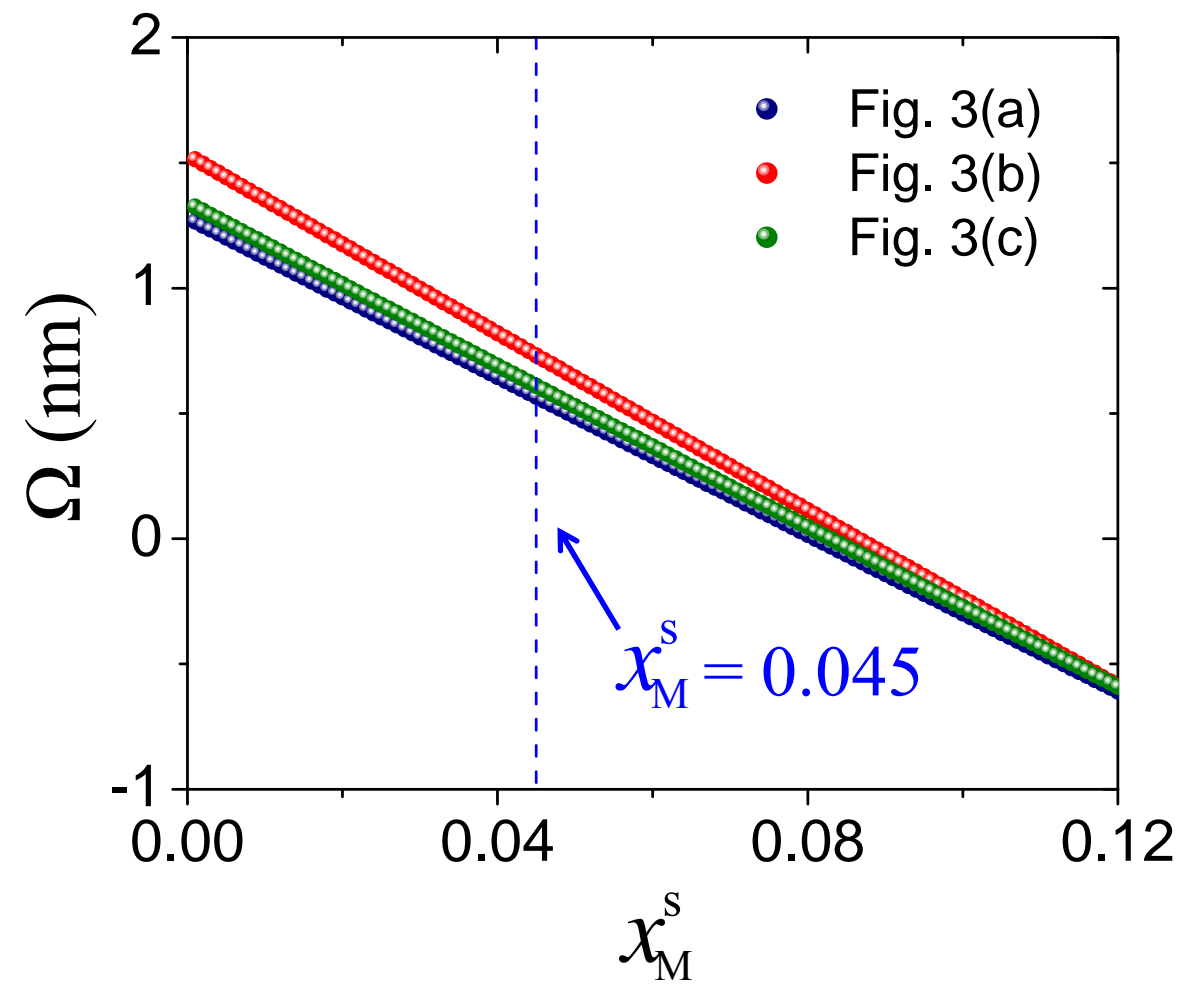

Figure S2: sets of $\left(x_{M}^{S} ; \Omega\right)$ pairs that reproduce the observed morphology of Si nanowires shown in Fig. 3. The vertical dashed line denotes the content of the catalyst atoms measured for these nanowires. Note the linear behavior of $\Omega v s . x_{M}^{S}$. 


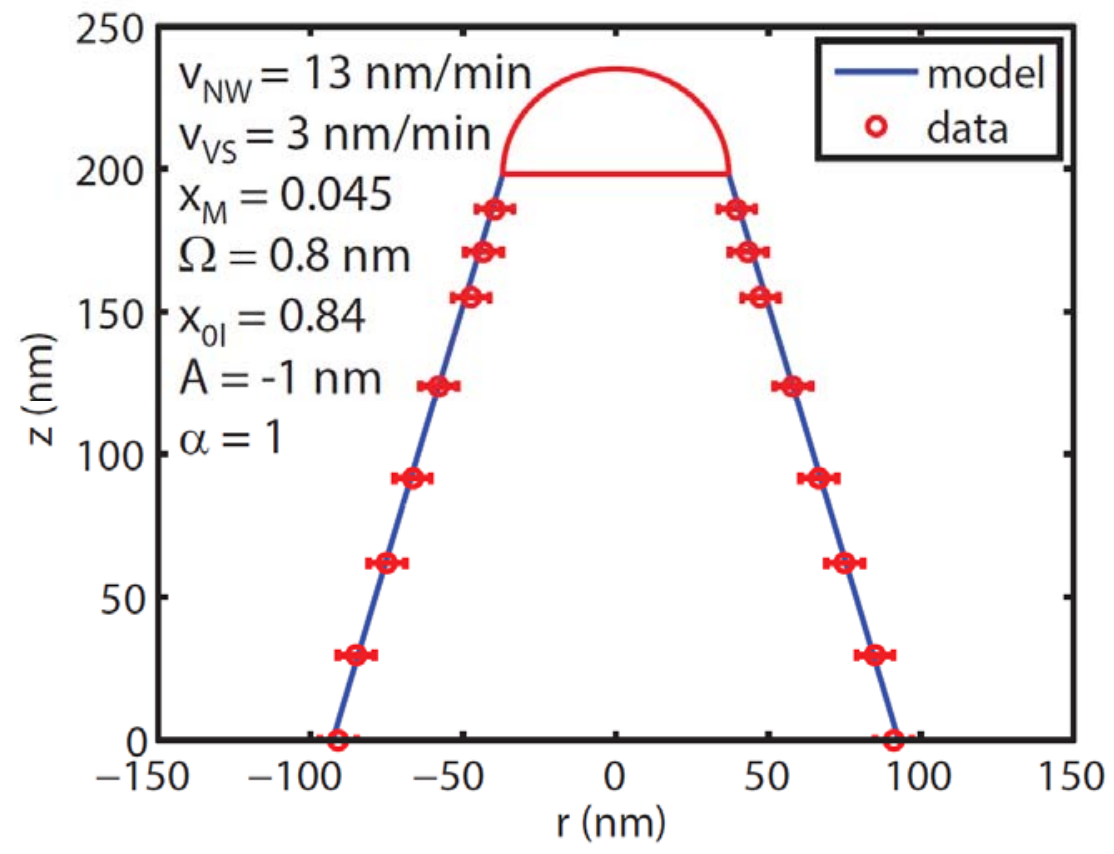

Figure S3: shape of a nanowire grown at $520^{\circ} \mathrm{C}$ using the same parameters as those obtained for nanowires grown at $470^{\circ} \mathrm{C}\left(x_{s}=0.045, \Omega=0.8 \mathrm{~nm}\right.$ and $\left.A=-1 \mathrm{~nm}\right)$ while also including vapor solid growth $(3 \mathrm{~nm} / \mathrm{min})$. The nanowire growth velocity was taken directly from reference 45 . 


\section{Effect of the nanodroplet size on the nanowire growth}

The variation in the nanodroplet size can possibly induce a change in its equilibrium composition. ${ }^{37}$ This effect is reduced in the model to the parameter A, which describes the shift in the equilibrium composition of the nanodroplet as a function of its radius. Fig. 2(c) displays the effect of this phenomenon on the nanowire morphology. By fitting the data reported in reference 37 , we found that the typical values of $\mathrm{A}$ are negative and on the order of a few nanometers. Fig. 2(c) shows a set of nanowires simulated at $\mathrm{x}_{\mathrm{M}}^{\mathrm{s}}=0.045, \Omega=0.5 \mathrm{~nm}$, and A varying between $-5 \mathrm{~nm}$ and $0 \mathrm{~nm}$. The obtained result indicates that $\mathrm{A}$, and thus supercooling, has a rather limited effect on the nanowire morphology. This is not surprising because the nanowire radius considered here $(50 \mathrm{~nm})$ is sufficiently large for the nanodroplet to behave practically as a bulk alloy. Our calculations show that the shape of nanowires becomes more sensitive to the shift in the nanodroplet equilibrium composition for a radius below $5 \mathrm{~nm}$ thus the size effect cannot be significant for the nanowires investigated here. It is noteworthy that this proposed model is not valid for nanowire radii below a critical value of $-\frac{A \beta^{\frac{1}{3}}}{\hat{\mathrm{x}}_{M}^{1}} \approx 1.3 \mathrm{~nm}$. This limit comes from the approximations used in the thermodynamic calculations of sizedependent phase diagram. 


\section{Modeling catalyst atom trapping in a growing nanowire}

The growth of a single bilayer in the VLS growth mode can be divided in two steps: the incubation period and the crystallization at the nanodroplet-nanowire interface. During the incubation, the concentration of the semiconductor in the nanodroplet increases until it reaches a critical value. Beyond this critical concentration, nucleation and growth take place. To model this phenomenon, we will consider the mass transfer during these two steps. The proposed model begins right after the deposition of a monolayer so the exact metal concentration in the droplet is unknown, but we assume that it will be the same after the subsequent growth of the next layer. In the beginning $(t=0)$, we have:

$$
\begin{gathered}
V_{C A T}^{0}=V_{M}^{0}+V_{S}^{0} \\
V_{M}^{0}=\hat{x}_{M 0}^{l} V_{C A T}^{0} \\
V_{S}^{0}=\hat{x}_{S 0}^{l} V_{C A T}^{0}
\end{gathered}
$$

where $V_{C A T}^{0}$ is the nanodroplet initial volume, $V_{M}^{0}$ and $V_{S}^{0}$ are the initial effective volumes of metal and semiconductor in the nanodroplet, respectively. $\hat{x}_{M 0}^{l}$ and $\hat{x}_{S 0}^{l}$ are metal and semiconductor equilibrium concentrations, respectively. At the end of the incubation period $\left(t=\tau_{I}\right)$, the volume of the catalyst can be expressed as:

$$
V_{C A T}^{I}=V_{C A T}^{0}+\tau_{I}\left(\Phi_{S}-\Phi_{M}\right)
$$

and metal and semiconductor volumes in the nanodroplet:

$$
\begin{gathered}
V_{M}^{I}=\hat{x}_{M 0}^{l} V_{C A T}^{0}-\tau_{\mathrm{I}} \Phi_{M} \\
V_{S}^{I}=\hat{x}_{S 0}^{l} V_{C A T}^{0}+\tau_{\mathrm{I}} \Phi_{S}
\end{gathered}
$$


where $\Phi_{S}$ is the flux of semiconductor atoms absorbed by the nanodroplet after the breakdown of precursor molecules, $\Phi_{M}$ is the flux of diffusing metal atoms. After the growth of a bilayer $\left(t=\tau_{I}+\tau_{B L}=\tau\right)$, the nanodroplet can be written as:

$$
V_{C A T}^{B L}=V_{C A T}^{0}+\tau\left(\Phi_{S}-\Phi_{M}\right)-V_{B L}
$$

and metal and semiconductor volumes in the nanodroplet:

$$
\begin{gathered}
V_{M}^{B L}=\hat{x}_{M 0}^{l} V_{C A T}^{0}-\tau \Phi_{M}-x_{M}^{S} V_{B L} \\
V_{S}^{B L}=\hat{x}_{S 0}^{l} V_{C A T}^{0}+\tau \Phi_{S}-x_{S}^{S} V_{B L}
\end{gathered}
$$

where $\Phi_{S}$ is the flux of semiconductor atoms absorbed by the nanodroplet after the breakdown of precursor molecules, $\Phi_{M}$ is the flux of diffusing metal atoms. $V_{B L}$ is the volume of a bilayer. $x_{M}^{S}=1-x_{S}^{S}$ is the fraction of metal atoms trapped in the grown bilayer. We now want to obtain the excess of semiconductor when the nucleation takes place:

$$
\begin{gathered}
x_{S}^{l}-\hat{x}_{S}^{l}=\frac{V_{S}^{I}}{V_{C A T}^{I}}-\hat{x}_{S}^{l} \\
=\frac{\hat{x}_{S 0}^{l} V_{C A T}^{0}+\tau_{\mathrm{I}} \Phi_{S}}{V_{C A T}^{0}+\tau_{\mathrm{I}}\left(\Phi_{S}-\Phi_{M}\right)}-\hat{x}_{S}^{l} \\
=\frac{\left(\hat{x}_{S 0}^{l} V_{C A T}^{0}+\tau_{\mathrm{I}} \Phi_{S}\right)-\hat{x}_{S}^{l}\left(V_{C A T}^{0}+\tau_{\mathrm{I}}\left(\Phi_{S}-\Phi_{M}\right)\right)}{V_{C A T}^{0}+\tau_{\mathrm{I}}\left(\Phi_{S}-\Phi_{M}\right)} \\
=\frac{V_{C A T}^{0}\left(\hat{x}_{S 0}^{l}-\hat{x}_{S}^{l}\right)+\tau_{\mathrm{I}}\left(\Phi_{S} \hat{x}_{M}^{l}+\hat{x}_{S}^{l} \Phi_{M}\right)}{V_{C A T}^{0}+\tau_{\mathrm{I}}\left(\Phi_{S}-\Phi_{M}\right)}
\end{gathered}
$$


The chemical potential of the nanodroplet is equal to $\mu_{C A T}=\mu_{0}+\mu_{C}$ and can be expressed to the lowest order as:

$$
\mu_{C A T}=\mu_{0}+\left(1-\hat{x}_{S}^{l}\right) g^{\prime \prime}\left(x_{S}^{l}-\hat{x}_{S}^{l}\right)
$$

From the equations above, we obtain:

$$
\mu_{C}=\left(1-\hat{x}_{S}^{l}\right) g^{\prime \prime} \frac{V_{C A T}^{0}\left(\hat{x}_{S 0}^{l}-\hat{x}_{S}^{l}\right)+\tau_{\mathrm{I}}\left(\Phi_{S} \hat{x}_{M}^{l}+\hat{x}_{S}^{l} \Phi_{M}\right)}{V_{C A T}^{0}+\tau_{\mathrm{I}}\left(\Phi_{S}-\Phi_{M}\right)}
$$

To continue the development, we can now consider the general case that, after the formation of a bilayer, the nanodroplet does not go back to the equilibrium, but it remains at an intermediate chemical potential: $\mu_{C A T}=\mu_{0}+\mu_{B L}$, corresponding to the concentration $\hat{x}_{S 0}^{l}$. The excess catalyst concentration of the nanodroplet can then be written as:

$$
\begin{gathered}
\hat{x}_{S 0}^{l}-\hat{x}_{S}^{l}=\frac{V_{S}^{B L}}{V_{C A T}^{B L}}-\frac{V_{S}^{0}}{V_{C A T}^{0}} \\
=\frac{\hat{x}_{S 0}^{l} V_{C A T}^{0}+\tau \Phi_{S}-x_{S}^{S} V_{B L}}{V_{C A T}^{0}+\tau\left(\Phi_{S}-\Phi_{M}\right)-V_{B L}}-\frac{\hat{x}_{S}^{l} V_{C A T}^{0}}{V_{C A T}^{0}} \\
=\frac{\hat{x}_{S 0}^{l} V_{C A T}^{0}+\tau \Phi_{S}-x_{S}^{S} V_{B L}-\hat{x}_{S}^{l}\left(V_{C A T}^{0}+\tau\left(\Phi_{S}-\Phi_{M}\right)-V_{B L}\right)}{V_{C A T}^{0}+\tau\left(\Phi_{S}-\Phi_{M}\right)-V_{B L}} \\
=\frac{V_{C A T}^{0}\left(\hat{x}_{S 0}^{l}-\hat{x}_{S}^{l}\right)+\tau\left(\hat{x}_{M}^{l} \Phi_{S}+\hat{x}_{S}^{l} \Phi_{M}\right)+V_{B L}\left(\hat{x}_{S}^{l}-x_{S}^{S}\right)}{V_{C A T}^{0}+\tau\left(\Phi_{S}-\Phi_{M}\right)-V_{B L}}
\end{gathered}
$$

We can now write

$$
\mu_{B L}=\left(1-\hat{x}_{S}^{l}\right) g^{\prime \prime}\left(\hat{x}_{S 0}^{l}-\hat{x}_{S}^{l}\right)
$$




$$
\mu_{B L}=\left(1-\hat{x}_{S}^{l}\right) g^{\prime \prime} \frac{V_{C A T}^{0}\left(\hat{x}_{S 0}^{l}-\hat{x}_{S}^{l}\right)+\tau\left(\hat{x}_{M}^{l} \Phi_{S}+\hat{x}_{S}^{l} \Phi_{M}\right)+V_{B L}\left(\hat{x}_{S}^{l}-x_{S}^{S}\right)}{V_{C A T}^{0}+\tau\left(\Phi_{S}-\Phi_{M}\right)-V_{B L}}
$$

from which we can extract the concentration of semiconductor in the nanowire:

$$
x_{S}^{S}=\hat{x}_{S}^{l}+\frac{V_{C A T}^{0}\left(\hat{x}_{S 0}^{l}-\hat{x}_{S}^{l}\right)+\tau\left(\hat{x}_{M}^{l} \Phi_{S}+\hat{x}_{S}^{l} \Phi_{M}\right)}{V_{B L}}-\frac{\mu_{B L}\left(V_{C A T}^{0}+\tau\left(\Phi_{S}-\Phi_{M}\right)-V_{B L}\right)}{V_{B L}\left(1-\hat{x}_{S}^{l}\right) g^{\prime \prime}}
$$

Since we are mainly interested in the metal incorporation we can rewrite this equation as:

$$
x_{M}^{S}=\hat{x}_{M}^{l}-\frac{V_{C A T}^{0}\left(\hat{x}_{S 0}^{l}-\hat{x}_{S}^{l}\right)+\tau\left(\hat{x}_{M}^{l} \Phi_{S}+\hat{x}_{S}^{l} \Phi_{M}\right)}{V_{B L}}+\frac{\mu_{B L}\left(V_{C A T}^{0}+\tau\left(\Phi_{S}-\Phi_{M}\right)-V_{B L}\right)}{V_{B L}\left(1-\hat{x}_{S}^{l}\right) g^{\prime \prime}}
$$

For a sufficiently important trapping, a general model should also consider the change in the radius. In this case, a given bilayer (i) should correspond to different volumes $V_{C A T}^{I, i}$ and $V_{B L}^{j}$. Thus, the volume of the catalyst after the $i^{\text {th }}$ the incubation cycle is:

$$
V_{C A T}^{I, i}=V_{C A T}^{0}+\sum_{j=1}^{i-1} \tau^{j}\left(\Phi_{S}^{j}-\Phi_{M}^{j}\right)+\tau_{I}^{i}\left(\Phi_{S}^{i}-\Phi_{M}^{i}\right)-\sum_{j=1}^{i-1} V_{B L}^{j}
$$

and the volume of the catalyst after the growth of the $i^{\text {th }}$ layer is:

$$
V_{C A T}^{B L, i}=V_{C A T}^{0}+\sum_{j=1}^{i} \tau^{j}\left(\Phi_{S}^{j}-\Phi_{M}^{j}\right)-\sum_{j=1}^{i} V_{B L}^{j}
$$


The volume of semiconductor in the nanodroplet after the $i^{\text {th }}$ incubation is:

$$
V_{S}^{I, i}=\hat{x}_{S 0}^{l} V_{C A T}^{0}+\sum_{j=1}^{i-1} \tau^{j} \Phi_{S}^{j}+\tau_{I}^{i} \Phi_{S}^{i}-\sum_{j=1}^{i-1} x_{S}^{s, j} V_{B L}^{j}
$$

The volume of semiconductor in the nanodroplet after the growth of the $i^{\text {th }}$ layer is:

$$
V_{S}^{B L, i}=\hat{x}_{S 0}^{l} V_{C A T}^{0}+\sum_{j=1}^{i} \tau^{j} \Phi_{S}^{j}-\sum_{j=1}^{i} x_{S}^{s, j} V_{B L}^{j}
$$

The excess of semiconductor in the droplet after the growth of the $i^{\text {th }}$ layer is:

$$
\begin{gathered}
x_{S}^{l, i}-\hat{x}_{S}^{l}=\frac{V_{S}^{B L, i}}{V_{C A T}^{B L, i}}-\frac{V_{S}^{0}}{V_{C A T}^{0}} \\
=\frac{\hat{x}_{S 0}^{l} V_{C A T}^{0}+\sum_{j=1}^{i} \tau^{j} \Phi_{S}^{j}-\sum_{j=1}^{i} x_{S}^{S, j} V_{B L}^{j}}{V_{C A T}^{0}+\sum_{j=1}^{i} \tau^{j}\left(\Phi_{S}^{j}-\Phi_{M}^{j}\right)-\sum_{j=1}^{i} V_{B L}^{j}}-\hat{x}_{S}^{l} \\
=\frac{V_{C A T}^{0}\left(\hat{x}_{S 0}^{l}-\hat{x}_{S}^{l}\right)+\sum_{j=1}^{i} \tau^{j} \Phi_{S}^{j}-\sum_{j=1}^{i} x_{S}^{s, j} V_{B L}^{j}-\hat{x}_{S}^{l}\left(\sum_{j=1}^{i}\left(\tau^{j}\left(\Phi_{S}^{j}-\Phi_{M}^{j}\right)-V_{B L}^{j}\right)\right)}{V_{C A T}^{0}+\sum_{j=1}^{i} \tau^{j}\left(\Phi_{S}^{j}-\Phi_{M}^{j}\right)-\sum_{j=1}^{i} V_{B L}^{j}} \\
=\frac{V_{C A T}^{0}\left(\hat{x}_{S 0}^{l}-\hat{x}_{S}^{l}\right)+\sum_{j=1}^{i}\left\{\tau^{j} \Phi_{S}^{j}-x_{S}^{S, j} V_{B L}^{j}-\hat{x}_{S}^{l}\left(\tau^{j}\left(\Phi_{S}^{j}-\Phi_{M}^{j}\right)-V_{B L}^{j}\right)\right\}}{V_{C A T}^{0}+\sum_{j=1}^{i}\left(\tau^{j}\left(\Phi_{S}^{j}-\Phi_{M}^{j}\right)-V_{B L}^{j}\right)} \\
x_{S}^{l, i}-\hat{x}_{S}^{l}=\frac{V_{C A T}^{0}\left(\hat{x}_{S 0}^{l}-\hat{x}_{S}^{l}\right)+\sum_{j=1}^{i}\left\{\left(x_{M}^{s, j}-\hat{x}_{M}^{l}\right) V_{B L}^{j}+\tau^{j}\left(\hat{x}_{M}^{l} \Phi_{S}^{j}+\hat{x}_{S}^{l} \Phi_{M}^{j}\right)\right\}}{V_{C A T}^{0}+\sum_{j=1}^{i}\left(\tau^{j}\left(\Phi_{S}^{j}-\Phi_{M}^{j}\right)-V_{B L}^{j}\right)}
\end{gathered}
$$

We can now write the chemical potential after the growth of the $i^{\text {th }}$ layer as: 


$$
\begin{gathered}
\mu_{0}+\mu_{B L}^{i}=\mu_{0}+\left(1-\hat{x}_{S}^{l}\right) g^{\prime \prime}\left(x_{S i}^{l, i}-\hat{x}_{S}^{l}\right) \\
\mu_{B L}=\left(1-\hat{x}_{S}^{l}\right) g^{\prime \prime} \frac{V_{C A T}^{0}\left(\hat{x}_{S 0}^{l}-\hat{x}_{S}^{l}\right)+\sum_{j=1}^{i}\left\{\left(x_{M}^{S, j}-\hat{x}_{M}^{l}\right) V_{B L}^{j}+\tau^{j}\left(\hat{x}_{M}^{l} \Phi_{S}^{j}+\hat{x}_{S}^{l} \Phi_{M}^{j}\right)\right\}}{V_{C A T}^{0}+\sum_{j=1}^{i}\left(\tau^{j}\left(\Phi_{S}^{j}-\Phi_{M}^{j}\right)-V_{B L}^{j}\right)}
\end{gathered}
$$

A uniform incorporation of metal atoms along the growth of the entire nanowire yields:

$$
\begin{array}{r}
\mu_{B L}=\left(1-\hat{x}_{S}^{l}\right) g^{\prime \prime} \frac{V_{C A T}^{0}\left(\hat{x}_{S 0}^{l}-\hat{x}_{S}^{l}\right)+x_{M}^{s} \sum_{j=1}^{i} V_{B L}^{j}-\hat{x}_{M}^{l} \sum_{j=1}^{i} V_{B L}^{j}+\sum_{j=1}^{i}\left\{\tau^{j}\left(\hat{x}_{M}^{l} \Phi_{S}^{j}+\hat{x}_{S}^{l} \Phi_{M}^{j}\right)\right\}}{V_{C A T}^{0}+\sum_{j=1}^{i}\left(\tau^{j}\left(\Phi_{S}^{j}-\Phi_{M}^{j}\right)-V_{B L}^{j}\right)} \\
x_{M}^{S}=\hat{x}_{M}^{l}+\frac{\mu_{B L}}{\hat{x}_{M}^{l} g^{\prime \prime}}\left(\frac{V_{C A T}^{0}+\sum_{j=1}^{i}\left(\tau^{j}\left(\Phi_{S}^{j}-\Phi_{M}^{j}\right)-V_{B L}^{j}\right)}{\sum_{j=1}^{i} V_{B L}^{j}}\right) \\
-\frac{V_{C A T}^{0}\left(\hat{x}_{S 0}^{l}-\hat{x}_{S}^{l}\right)+\sum_{j=1}^{i}\left\{\tau^{j}\left(\hat{x}_{M}^{l} \Phi_{S}^{j}+\hat{x}_{S}^{l} \Phi_{M}^{j}\right)\right\}}{\sum_{j=1}^{i} V_{B L}^{j}}
\end{array}
$$

